For Internal Distribution Only

Accelerator Division

Alternating Gradient Synchrotron Department

BROOKHAVEN NATIONAL LABORATORY

Upton, New York 11973

Accelerator Division

Technical Note

AGS/AD/Tech. Note No. 448

Heavy Ion Parameters for 1996

C.J. Gardner

October 10, 1996 


\title{
Heavy Ion Parameters for 1996
}

\author{
C. J. Gardner
}

October 10,1996

This is the 1996 version of the note on heavy ion parameters put out last year [1]. Ions of Iron and Gold will again be accelerated in the Tandem, Booster, and AGS for the 1996 HIP (Heavy Ion Physics) run. For the first part of the run, NASA again requires Iron ions at $1 \mathrm{GeV}$ kinetic energy per nucleon to bombard various biological samples. They have also expressed interest in acquiring some data with ions at $600 \mathrm{MeV}$ per nucleon. The iron ions will be extracted by slow extraction from the AGS and transported to experimental area A3. For the remainder of the run, Gold ions will be delivered to the experimental areas and to the ATR (AGS To RHIC) line. The experiments require Slow Extracted Beam while the ATR line requires a single bunch kicked out of the AGS with the Fast Extracted Beam setup commissioned last year. All experiments except two require Gold ions at the same momentum delivered for the past two years, namely $11.7 \mathrm{GeV} / \mathrm{c}$ per nucleon. (This momentum has been verified by measurements of frequency and radius in the AGS at extraction [2].) The other two experiments require Gold ions with kinetic energies of 6 and 8 $\mathrm{GeV}$ per nucleon. The ATR line and RHIC require gold ions with momentum $11.2 \mathrm{GeV} / \mathrm{c}$ per nucleon. Following is a summary of the various ion parameters during injection, acceleration, and extraction in the Tandem, Booster and AGS.

\section{Charge State and Acceleration Overview}

Negative ions enter the Tandem with charge minus one and are accelerated from ground potential to the center terminal where they are stripped of $1+Q_{t}$ electrons thereby becoming positive ions (with charge $Q_{t}$ ) which are then accelerated back to ground potential. A foil located downstream of 
the Tandem and upstream of the first $90^{\circ}$ bend in the TTB (Tandem To Booster) line may be inserted to allow additional stripping of the ions before they enter the Booster. The charge state after this stripping is $Q_{b}$. Ions with this charge are transported down the TTB line and injected into the Booster where they are captured and undergo further acceleration. The harmonic of the accelerating voltage is $h=8$, and, for the case in which the ions are to be slowly extracted from the AGS, the bunches are simply kicked out of the Booster after acceleration at this harmonic. (The F3 kicker in the Booster and the A5 kicker in the AGS cannot stay on long enough for all 8 bunches to be extracted from the Booster and injected into the AGS; typically only 5 of the 8 bunches are cleanly transfered to the AGS. This situation eventually will be remedied with modified power supplies for the kicker magnets.) For the case in which a single bunch is to be delivered to RHIC, an accelerating voltage with harmonic $h=4$ is turned on in the Booster just before extraction and the bunches are allowed to rotate $90^{\circ}$ in the $h=4$ buckets-a process we call a pseudo-merge - after which they are kicked out of the Booster.

After extraction from the Booster, the ions pass through a foil in the BTA (Booster To AGS) line and emerge with charge $Q_{a}$. Ions with this charge are transported down the BTA line and injected into the AGS where they are again accelerated. If the bunches have undergone a pseudo-merge before extraction from the Booster, they are transfered into Rf buckets at harmonic $h_{a}=16$ in the AGS and accelerated at this harmonic; otherwise they are first allowed to debunch and are then captured and accelerated to full energy at harmonic $h_{a}=12$. At harmonic $h_{a}=16$ the bunches cannot be accelerated to full energy; after acceleration at $h_{a}=16$ they must be merged and then accelerated to full energy at harmonic $h_{a}=8$. (This year we will do an additional merge before extraction with an accelerating voltage at harmonic $h_{a}=4$.)

After extraction from the AGS, the ions are either transported to fixed-target experiments in the experimental hall or they are sent down the ATR line where they pass through a final foil which strips away the remaining electrons. The charge of the fully stripped ion is $Q_{r}$. The nominal charge states for the ions of the $1996 \mathrm{HIP}$ run are summarized in the following table. (Note that the subscripts $t, b, a$, and $r$ are used to denote the charge states in the tandem, booster, ags, and rhic respectively.) The number of nucleons, $n$, and atomic mass, $a$, are also included. 


\begin{tabular}{|c|c|c|c|c|c|c|}
\hline \multicolumn{6}{|c|}{ Table I: Charge States and Atomic Parameters } \\
\hline Atom & $n$ & $a$ & $Q_{t}$ & $Q_{b}$ & $Q_{a}$ & $Q_{r}$ \\
\hline Iron & 56 & 55.93434 & +10 & +10 & +26 & +26 \\
Gold & 197 & $\mathbf{1 9 6 . 9 6 6 5 4 1}$ & +12 & +32 & +77 & +79 \\
\hline
\end{tabular}

\section{Acceleration in Tandem}

Negative ions of iron oxide $\left(\mathrm{FeO}^{-}\right)$or gold $\left(\mathrm{Au}^{-}\right)$enter the Tandem with charge minus one and mass $m_{s}$, having been accelerated through a potential difference of

$$
V_{s}=130 \mathrm{keV}
$$

in the ion source. Neglecting the atomic binding energy, the mass of the iron oxide ion is

$$
m_{s}=a u+b u+m_{e}
$$

where $a=55.93493$ is the atomic mass of iron $\left(\mathrm{Fe}^{56}\right), b=15.99491$ is the atomic mass of oxygen $\left(\mathrm{O}^{16}\right), u=931.49432 \mathrm{MeV} / c^{2}$ is the unified atomic mass unit, and $m_{e} c^{2}=.5110034 \mathrm{MeV}$ is the electron mass. The mass of the gold ions $\left(\mathrm{Au}^{-}\right)$is

$$
m_{s}=a u+m_{e}
$$

where $a=196.966541$. The part of the negative ion consisting of a neutral atom of iron or gold, minus $Q_{t}$ electrons, has mass

$$
m_{t}=a u-Q_{t} m_{e}
$$

and gains energy

$$
W_{s}=V_{s} m_{t} / m_{s}
$$

in the ion source. Upon acceleration from the "tank" potential to the center terminal of the Tandem, this part of the negative ion gains energy

$$
W_{f}=V_{t} m_{t} / m_{s}
$$

where $V_{t}$ is the terminal voltage. In the center terminal the ions pass through a thin foil and emerge as individual atoms with various charge states. Upon acceleration back to "tank" potential, the iron or gold ions with charge $Q_{t}$ gain additional energy $Q_{t} V_{t}$ so that the total energy gained by each of these ions due to the accelerating voltage in the Tandem is

$$
W_{t}=W_{f}+Q_{t} V_{t}=\left(m_{t} / m_{s}+Q_{t}\right) V_{t}
$$


Assuming each ion loses energy $\delta W$ as it passes through the foil in the center terminal, the total kinetic energy of the ion emerging from the Tandem will be $W_{s}+W_{t}-\delta W$. For the 2 microgram foils typically used in the terminal, $\delta W$ is approximately $180 \mathrm{keV}$ (as per Peter Thieberger).

\section{Booster Injection}

The momentum of ions transported down the TTB line and injected into the Booster is determined by the setting of the magnetic field (as measured by NMR probes) in the $90^{\circ}$ bends of the TTB line. The Tandem voltage required to give the desired momentem is then obtained by adjusting the voltage so that the ion beam is centered in slits located downstream of the bends. If we let $p$ be the momentum of the ions injected into the booster, then their energy is

$$
E=\sqrt{p^{2} c^{2}+m^{2} c^{4}}
$$

where the mass is

$$
m=a u-Q_{b} m_{e} .
$$

(Putting in numbers we find that the masses of the iron and gold ions injected into the booster are 52.0979596 and $183.4568621 \mathrm{GeV} / c^{2}$.) The kinetic energy is then

$$
W=E-m c^{2}
$$

and

$$
W_{s}+W_{t}-\delta W=W
$$

where $W_{s}$ and $W_{t}$ are given by (5) and (7). The Tandem voltage is therefore

$$
V_{t}=W_{t} /\left(m_{t} / m_{s}+Q_{t}\right)=\left(W-W_{s}+\delta W\right) /\left(m_{t} / m_{s}+Q_{t}\right) .
$$

It is convenient to parameterize the momentum and energy in terms of the kinetic energy defined by (10). Thus using (8) and (10) we have

$$
c p=\sqrt{W^{2}+2 m c^{2} W}, \quad E=m c^{2}+W .
$$

We can then derive all other injection parameters from $c p$ and $E$. Thus the rigidity of the ion beam in units of $\mathrm{Tm}$ is

$$
B \rho=k p / Q
$$


where $k=3.33564095 \times 10^{-3}, p$ is the momentum in units of $\mathrm{MeV} / \mathrm{c}$, and $Q$ is the ion charge. The inflector voltage, $V_{I}$, required to bring the beam into the acceptance region of the Booster is [3]

$$
e V_{I}=\frac{D}{R} c^{2} p^{2} /(Q E)
$$

where $D=0.017 \mathrm{~m}$ and $R=8.74123 \mathrm{~m}$. The velocity and revolution frequency of the ion as it enters the Booster are

$$
v=c \beta=c^{2} p / E, \quad f=v / C, \quad C=2 \pi R_{a} / 4=\pi R_{a} / 2
$$

where $C$ is the Booster circumference and $R_{a}=128.454$ meters is the radius of the AGS. The frequency of the accelerating voltage in the Booster is $h f$, where $h=8$ for the 1996 run. The following table summarizes the various Tandem and Booster injection parameters. The nominal injection parameters for iron and gold are those corresponding to kinetic energies $W=126.8$ and $182.13 \mathrm{MeV}$ respectively.

\begin{tabular}{|c|c|c|c|c|c|c|}
\hline \multicolumn{7}{|c|}{ Table II: Tandem and Booster Injection Parameters } \\
\hline Ion & $W(\mathrm{MeV})$ & $V_{t}(\mathrm{MV})$ & $c p(\mathrm{MeV} / n)$ & $B \rho(\mathrm{Tm})$ & $V_{I}(\mathrm{kV})$ & $h f(\mathrm{kHz})$ \\
\hline \multirow{3}{*}{$\mathrm{Fe}^{10+}$} & 125.8 & 11.680 & 64.69037 & 1.20839 & 48.872 & 824.523 \\
& 126.8 & 11.773 & 64.94729 & 1.21319 & 49.260 & 827.782 \\
& 127.8 & 11.865 & 65.20320 & 1.21797 & 49.648 & 831.027 \\
\hline \multirow{3}{*}{$\mathrm{Au}^{32+}$} & 181.13 & 13.937 & 41.39216 & .849990 & 22.006 & 527.795 \\
& 182.13 & 14.014 & 41.50632 & .852334 & 22.127 & 529.247 \\
& 183.13 & 14.091 & 41.62017 & .854672 & 22.248 & $\mathbf{5 3 0 . 6 9 6}$ \\
\hline
\end{tabular}

Note that the values of $W, V_{t}, V_{I}$, and $h f$ listed for iron differ slightly from those calculated in Ref. [1]. This is because that calculation used the weighted atomic mass of iron rather than the atomic mass of the most abundant isotope, $\mathrm{Fe}^{56}$, which is what is actually delivered by the Tandem. The calculation of the terminal voltage was also incorrect for both iron and gold. These errors have been corrected and the parameters listed in Table II have been adjusted so that the momentum $c p$ and magnetic rigidity $B \rho$ are the same as they were last year. 


\section{Booster Extraction}

The iron and gold ions are accelerated at harmonic $h=8$ to $h f=5.0 \mathrm{MHz}$ before being kicked out of the Booster into the BTA line. Since the maximum energy to which a given ion can be accelerated is ultimately limited by the maximum field of the Booster magnets, it is convenient to parameterize the Booster extraction parameters in terms of the magnetic rigidity $B \rho$. Thus we have

$$
p=Q_{b} B \rho / k, \quad E=\sqrt{p^{2} c^{2}+m^{2} c^{4}}, \quad W=E-m c^{2}, \quad f=c^{2} p /(E C)
$$

where $m$ is given by (9) and $k$ is defined by (14). We also define $P$ to be the momentum of a proton which has the same rigidity as the ion under consideration. Thus

$$
P=B \rho / k=p / Q_{b}
$$

The following table summarizes the various Booster extraction parameters.

\begin{tabular}{|c|c|c|c|c|c|}
\hline \multicolumn{7}{|c|}{ Table III: Booster Extraction Parameters } \\
\hline Ion & $B \rho(\mathrm{Tm})$ & $c P(\mathrm{GeV})$ & $c p(\mathrm{MeV} / n)$ & $W(\mathrm{MeV} / n)$ & $h f(\mathrm{MHz})$ \\
\hline \multirow{3}{*}{$\mathrm{Fe}^{10+}$} & 7.6578 & 2.296 & 409.9555 & 86.3209 & 4.793051 \\
& 8.0578 & 2.416 & 431.3692 & 95.1431 & 5.000023 \\
& 8.4578 & 2.536 & 452.7830 & 104.3334 & 5.201614 \\
\hline \multirow{3}{*}{$\mathrm{Au}^{32+}$} & 8.4670 & 2.538 & 412.3196 & 87.1966 & 4.812133 \\
& 8.8670 & 2.658 & 431.7985 & 95.2372 & 4.999994 \\
& 9.2670 & 2.778 & 451.2774 & 103.5820 & 5.183411 \\
\hline
\end{tabular}

\section{Stripping in BTA}

After extraction from the Booster and before passing through the stripping foil in the BTA line, the ions have charge $Q_{b}$, mass $m_{b}=a u-Q_{b} m_{e}$, and energy $E_{b}=\sqrt{p_{b}^{2} c^{2}+m_{b}^{2} c^{4}}$, where $c p_{b}$ is given by $c p$ in Table III. The part of each of these ions consisting of a neutral atom of iron or gold, minus $Q_{a}$ electrons, has mass and energy

$$
m_{a}=a u-Q_{a} m_{e}, \quad E_{a}=E_{b} m_{a} / m_{b} .
$$

Upon passing through the foil, the ions lose energy $\delta E$ and those emerging with charge $Q_{a}$ then have energy and momentum

$$
E=E_{a}-\delta E, \quad c p=\sqrt{E^{2}-m_{a}^{2} c^{4}}
$$


The energy loss and stripping efficiencies in the BTA foil have been calculated and measured by Roser [4-6] for the case of $\mathrm{Au}^{33+}$ ions passing through various foils (Carbon, Aluminum, Copper) with momenta ranging from 330 to $760 \mathrm{MeV} / \mathrm{c}$ per nucleon. For the $1996 \mathrm{HIP}$ run, a 0.010 inch thick Carbon foil will be used to strip the $\mathrm{Fe}^{10+}$ ions to charge state $Q_{a}=26$ (fully stripped), and a 0.004 inch thick Carbon foil will be used to strip the $\mathrm{Au}^{32+}$ ions to charge state $Q_{a}=77$ (two remaining electrons). (The same foils were used in 1995.) The 0.004 and 0.010 inch thick foils occupy positions 3 and 6 respectively in the BTA foil holder.

Because it has been the source of confusion in the past, we note here that the foil holder in the BTA line consists of a rotatable circular array of eight equally spaced positions or slots, seven of which contain foils and one which is left open (or blank) for beams (protons) that do not require stripping. The location and characteristics of each of the seven foils are summarized in Table IV:

\begin{tabular}{|c|c|c|c|}
\hline \multicolumn{5}{|c|}{ Table IV: BTA Foils } \\
\hline Position & Foil & Thickness & Mass \\
\hline 1 & Blank & Inches & $\mathrm{mg} / \mathrm{cm}^{2}$ \\
\hline 2 & Carbon & .003 & 17 \\
3 & Carbon & .004 & 22 \\
4 & Carbon & .005 & 28 \\
5 & Carbon & .007 & 39 \\
6 & Carbon & .010 & 56 \\
\hline 7 & Copper & .001 & 23 \\
\hline 8 & Copper/Carbon & $.001 / .003$ & $23 / 17$ \\
\hline
\end{tabular}

The indicated thicknesses are those quoted by the manufacturer. For the Carbon foils they are good to about 0.0001 inches; for the Copper foil they are good to about 10 per cent. The mass per unit area has been calculated assuming densities of 2.2 and $8.9 \mathrm{~g} / \mathrm{cm}^{3}$ for Carbon and Copper.

To estimate the energy loss, $\delta E$, we employ the scaling law used in Refs. [4-6]:

$$
\left(\frac{d E}{d x}\right)_{N_{\mathrm{X} Z}}=\frac{Z^{2}}{N} \times\left(\frac{d E}{d x}\right)_{\text {Proton }}\left(p=\frac{p_{\mathrm{X}}}{N}\right) .
$$

Here ${ }^{N} X^{Z}$ denotes an ion of element $\mathrm{X}$ with $N$ nucleons and charge $Z, p$ and $p_{\mathrm{X}}$ are proton and ion momenta, and $d E / d x$ is the energy lost per unit length of the foil material. To apply (21) we need the value of $d E / d x$ in 
the foil material for protons with momentum $p=p_{\mathrm{X}} / N$. Consulting Table III we see that $p_{\mathrm{X}} / N$ is approximately $430 \mathrm{MeV} / \mathrm{c}$ for both iron and gold, and consulting Ref.[7] we find that $d E / d x$ for protons with $430 \mathrm{MeV} / \mathrm{c}$ momentum in Carbon is $6.2 \mathrm{MeV}$ per $\mathrm{g} / \mathrm{cm}^{2}$. For iron we have $Z^{2} / N=26^{2} / 56$, and for gold $Z^{2} / N=77^{2} / 197$. Using these numbers in (21) we then find that $\delta E=4.2 \mathrm{MeV}$ per nucleon for $\mathrm{Fe}^{26+}$ passing through foil 6 and also for $\mathrm{Au}^{77+}$ passing through foil 3 .

\section{AGS Injection}

It is convenient to parameterize the AGS injection parameters in terms of the energy and momentum given by (20). Thus we have

$$
\beta=c p / E, \quad f_{a}=c \beta /\left(2 \pi R_{a}\right), \quad W=E-m_{a} c^{2}
$$

where $f_{a}$ is the AGS revolution frequency, $R_{a}=128.454$ meters, and $m_{a}$ is given by (19). We also have

$$
B \rho=k p / Q_{a}, \quad P=B \rho / k=p / Q_{a}
$$

where $\boldsymbol{P}$ is the equivalent proton momentum. (The masses of the iron and gold ions in the AGS are 52.0897835 and $183.4338669 \mathrm{GeV} / \mathrm{c}^{2}$.)

Let us first assume that the ions lose no energy upon passing through the foil in BTA-i.e. we assume $\delta E=0$. We then obtain the parameters listed in Table V. The harmonic number at injection is taken to be $h_{a}=12$, although it is actually 16 for the case in which the bunches have undergone a pseudo-merge at Booster extraction.

\begin{tabular}{|c|c|c|c|c|c|}
\hline \multicolumn{7}{|c|}{ Table V: AGS Injection Parameters $(\delta E=0)$} \\
\hline Ion & $h_{a} f_{a}(\mathrm{MHz})$ & $B \rho(\mathrm{Tm})$ & $c P(\mathrm{GeV})$ & $c p(\mathrm{MeV} / n)$ & $W(\mathrm{MeV} / n)$ \\
\hline \multirow{3}{*}{$\mathrm{Fe}^{26+}$} & 1.797394 & 2.9448 & 0.883 & 409.8911 & 86.3073 \\
& 1.875009 & 3.0987 & 0.929 & 431.3015 & 95.1282 \\
& 1.950605 & 3.2525 & 0.975 & 452.7119 & 104.3170 \\
\hline \multirow{3}{*}{$\mathrm{Au}^{77+}$} & 1.804550 & 3.5183 & 1.055 & 412.2680 & 87.1857 \\
& 1.874998 & 3.6845 & 1.105 & 431.7444 & 95.2252 \\
& 1.943779 & 3.8507 & 1.154 & 451.2209 & 103.5690 \\
\hline
\end{tabular}

Note that the frequencies, $h_{a} f_{a}$, listed in Table $\mathrm{V}$ correspond to the frequencies, $h f$, listed in the last column of Table III, and in this case 
$(\delta E=0)$ we have $f_{a}=f / 4$. The ions do, of course, lose energy as they pass through the stripping foil and so the AGS revolution frequency, $f_{a}$, is in fact less than one fourth the Booster revolution frequency $f$. Using the estimated value of $\delta E=4.2 \mathrm{MeV}$ per nucleon obtained above for iron and gold, we obtain the parameters listed in Table VI.

\begin{tabular}{|c|c|c|c|c|c|}
\hline \multicolumn{5}{|c|}{ Table VI: AGS Injection Parameters $(\delta E=4.2 \mathrm{MeV}$ per nucleon) } \\
\hline Ion & $h_{a} f_{a}(\mathrm{MHz})$ & $B \rho(\mathrm{Tm})$ & $c P(\mathrm{GeV})$ & $c p(\mathrm{MeV} / n)$ & $W(\mathrm{MeV} / n)$ \\
\hline \multirow{3}{*}{$\mathrm{Fe}^{26+}$} & 1.758489 & $\mathbf{2 . 8 6 9 2}$ & $\mathbf{0 . 8 6 0}$ & 399.3619 & 82.1073 \\
& 1.838712 & $\mathbf{3 . 0 2 6 2}$ & 0.907 & 421.2198 & 90.9282 \\
& 1.916672 & 3.1829 & 0.954 & 443.0305 & 100.1170 \\
\hline \multirow{3}{*}{$\mathrm{Au}^{77+}$} & 1.765934 & 3.4288 & 1.028 & 401.7818 & 82.9857 \\
& 1.838739 & 3.5985 & 1.079 & 421.6627 & 91.0252 \\
& 1.909675 & 3.7678 & 1.130 & 441.5047 & 99.3690 \\
\hline
\end{tabular}

Comparing these parameters with the corresponding ones in Table $\mathrm{V}$, we see that when the Booster extraction frequency, $h f$, is $5 \mathrm{MHz}$, the AGS injection frequency, $h_{a} f_{a}$, is reduced from 1.875 to $1.839 \mathrm{MHz}$ (a reduction of $36 \mathrm{kHz}$ ) due to the energy loss in the foil.

\section{AGS Extraction}

It is convenient to parameterize the AGS extraction parameters in terms of the magnetic rigidity, $B \rho$, or the corresponding proton momentum $P=B \rho / k$. Thus we have

$$
\begin{gathered}
p=Q_{a} B \rho / k, \quad E=\sqrt{p^{2} c^{2}+m^{2} c^{4}}, \quad W=E-m c^{2}, \\
f_{a}=c^{2} p /\left(2 \pi R_{a} E\right), \quad \gamma=\frac{E}{m c^{2}}=1+\frac{W}{m c^{2}}
\end{gathered}
$$

where $m$ is given by (19) and $2 \pi R_{a}$ is the AGS circumference. Table VII summarizes the various parameters for ions of iron and gold. The harmonic number at extraction is taken to be $h_{a}=12$.

Note that for $\mathrm{Au}^{77+}$ ions with $W=6$ and $8 \mathrm{GeV}$ per nucleon at extraction, we have $\gamma=7.44$ and 9.59 which are both somewhat close to the nominal gamma-transition of 8.5. The concern here is that the time required for the beam to debunch becomes infinite as the gamma of the 
ions approaches gamma-transition (good debunching is required for a uniform SEB spill). The debunch time is proportional to $1 / \eta$, where

$$
\eta=\frac{1}{\gamma_{t}^{2}}-\frac{1}{\gamma^{2}}
$$

and $\gamma_{t} \approx 8.5$. For $\gamma=7.44$ and 9.59 we find $1 / \eta=-237$ and 337. These are to be compared with $1 / \eta=133$ for the case of $\mathrm{Au}^{77+}$ ions with momentum $11.7 \mathrm{GeV} / \mathrm{c}$ per nucleon which have a gamma of 12.6.

\begin{tabular}{|c|c|c|c|c|c|}
\hline \multicolumn{6}{|c|}{ Table VII: AGS Extraction Parameters } \\
\hline Ion & $B \rho(\mathrm{Tm})$ & $c P(\mathrm{GeV})$ & $c p(\mathrm{GeV} / n)$ & $W(\mathrm{GeV} / n)$ & $h_{a} f_{a}(\mathrm{Mhz})$ \\
\hline \multirow{4}{*}{$\mathrm{Fe}^{26+}$} & 8.7290 & 2.6169 & 1.21499 & 0.60000 & 3.539215 \\
& 10.4813 & 3.1422 & 1.45888 & 0.80001 & 3.758376 \\
& 12.1507 & 3.6427 & 1.69125 & 1.00000 & 3.905595 \\
& 13.7679 & 4.1275 & 1.91634 & 1.19999 & 4.009910 \\
& 15.3493 & 4.6016 & 2.13646 & 1.39999 & 4.086785 \\
\hline \multirow{4}{*}{$\mathrm{Au}^{77+}$} & 23.7187 & 7.1107 & 2.77931 & 2.00000 & 4.226442 \\
& 41.3256 & 12.3891 & 4.84244 & 4.00001 & 4.377141 \\
& 58.6145 & 17.5722 & 6.86832 & 6.00002 & 4.416922 \\
& 75.8034 & 22.7253 & 8.88248 & 8.00001 & 4.433036 \\
& 92.9480 & 27.8651 & 10.89144 & 10.00003 & 4.441126 \\
\hline \multirow{3}{*}{$\mathrm{Au}^{77+}$} & $\mathbf{9 3 . 8 7 4 6}$ & 28.1429 & 11.00002 & 10.10822 & 4.441443 \\
& 95.5815 & 28.6546 & 11.20002 & 10.30752 & 4.442002 \\
& 97.2880 & 29.1662 & 11.39999 & 10.50681 & 4.442532 \\
\hline \multirow{3}{*}{$\mathrm{Au}^{77+}$} & 98.9948 & 29.6779 & 11.59999 & 10.70617 & 4.443036 \\
& 99.8484 & 29.9338 & 11.70001 & 10.80587 & 4.443278 \\
& 100.7017 & 30.1896 & 11.80000 & 10.90554 & 4.443514 \\
\hline
\end{tabular}

\section{Beam Intensities}

Listed in Table VIII are Beam Intensities recorded during the 1995 HIP run when we were running well with one load transfered from Booster to AGS per AGS cycle. (For part of the Gold run we ran with lower current from Tandem but with two Booster loads transfered to the AGS per AGS cycle instead of just one.) 


\begin{tabular}{|c|c|c|}
\hline \multicolumn{3}{|c|}{ Table VIII: 1995 Beam Intensities } \\
\hline & Iron & Gold \\
\hline End of TTB: & & \\
\hline Current & 35 micro-Amps & 36 micro-Amps \\
\hline Pulse Width & 700 micro-sec & 660 micro-sec \\
\hline Total Ions & $153 \times 10^{8}$ & $46 \times 10^{8}$ \\
\hline Booster: & & \\
\hline Early & $32 \times 10^{8}$ Ions & $19 \times 10^{8}$ Ions \\
\hline Late & $25 \times 10^{8}$ Tons & $13 \times 10^{8}$ Ions \\
\hline AGS: & & \\
\hline Early & $4.9 \times 10^{8}$ Ions & $5.4 \times 10^{8}$ Ions \\
\hline Late & $4.2 \times 10^{8}$ Ions & $3.9 \times 10^{8}$ Ions \\
\hline Extracted & $3.4 \times 10^{8}$ Ions & $2.8 \times 10^{8}$ Ions \\
\hline Beam Lines: & & \\
\hline A Line & $1.0 \times 10^{8}$ Ions & $69 \times 10^{6}$ Ions \\
\hline B Line & & $6.2 \times 10^{6}$ Ions \\
\hline C Line & & $39 \times 10^{6}$ Ions \\
\hline
\end{tabular}

\section{References}

1. C.J. Gardner, "Heavy Ion Parameters for 1995", AGS/AD/Tech. Note No. 417, September 1, 1995.

2. L. Ahrens, "AGS Extraction Radius Revisited-Au" ${ }^{77+}$ December 1995", AGS Studies Report No. 339, January 30, 1996.

3. C.J. Gardner, "Booster Inflector Specifications", Booster Tech. Note No. 159, February 28, 1990.

4. T. Roser, "BTA Stripping Foil Studies", AGS/AD/Tech. Note No. 367, October 2, 1992.

5. T. Roser, "Stripping Efficiencies for $277 \mathrm{MeV} / \mathrm{amu}$ Gold Beam on Copper Foils", Proceedings of the 1993 Particle Accelerator Conf., Washington, D.C.,May 17-20, 1993, pp.3207-3209.

6. L. Ahrens, H.C. Hseuh, and T. Roser, "Charge Exchange Studies with Gold Ions at the Brookhaven Booster and AGS", Fourth European Particle Accelerator Conf., London, England, June 27-July 1, 1994, pp. 2441-2443.

7. "Review of Particle Properties", Phys. Rev. D45, Part 2, June 1992, p. III.20 